\title{
The Distribution of the Stress Protein HSP70 in the Cerebellum of Patients with Schizophrenia
}

\author{
Makris Leonidas \\ Theodor Theohari Cozzika Foundation, Neurobiology Research Institute, Athens, Greece. \\ Email: lemak1972@yahoo.com
}

Received September $25^{\text {th }}, 2012$; revised October $24^{\text {th }}, 2012$; accepted November $2^{\text {nd }}, 2012$

\begin{abstract}
Data accumulated from neuro-imaging, clinical and morphological studies suggest that the cerebellum is involved in cognitive functions and thus may be important in the etiopathogenesis of schizophrenia, since patients show cognitive abnormalities. In the present study, we have attempted to localize cellular metabolic dysfunctions applying the immunohistochemical and Western blot method to demonstrate the expression of the stress protein HSP70, which is a marker of cellular metabolic dysfunction in the brain. We studied the post mortem brains' cerebellum of 12 normal controls and 10 schizophrenics. We have used the polyclonal antibody rabbit anti-HSP70 on paraffin sections as well as on nitrocellulose membranes. Bound antibody was detected using the indirect method of streptavidin-peroxidase-DAB. The results in the cerebellum of controls showed intense HSP70 immunoreaction in the synaptic glomeruli of the granular cell layer, in the cytoplasm and dendrites of Purkinje cells. In the same areas of the cerebellum of schizophrenics the HSP70 immunoreactivity was minimal. These results suggest that the reduced levels of HSP70 in the cerebellum are likely to contribute synergistically to the cognitive dysfunction in schizophrenia. This may suggest abnormality of protective neural mechanisms in such pathological conditions.
\end{abstract}

Keywords: Biological Marker; Post Mortem; Heat Shock Protein; Cerebellum; Schizophrenia

\section{Introduction}

Data accumulated from neuroimaging, clinical and morphological studies, in controls, suggest that the cerebellum is involved not only in motor, but also in cognitive functions [1]. The cerebellum is thus important in the etiopathology of schizophrenia since neuroimaging, clinical, functional and cognitive studies [2] indicate that schizophrenic patients show abnormalities compared to controls. However, in schizophrenia, changes in expression of some proteins showed to influence the regulation of synaptic function [3]. Another study indicates the dysfunction of synapses in schizophrenia [4]. In particular, constitutive proteins of functional synapses such as synaptophysin and complexin I and II presented a reduced expression leading to damage of specific synapses. Additionaly, functional and constitutive cerebellar anomalies have been mentioned widely in diseases such as schizophrenia, anxiety, depression and mania [5].

It is believed that cells maintain the ability to respond to cellular stresses (variety of stimuli such as heat, hypoxia, radiation, viral infections, etc.) by increasing the expression of proteins referred to as Heat Shock Proteins (HSPs). The most studied member of HSP family is HSP70 which in normal functioning cells have been shown to participate in protein folding, molecular chaperoning and protein translocation [6]. The overexpression of HSP70 seems to function, as part of a mechanism responsible for cellular protection, in animal models after cerebral ischemia followed by cardiac arrest [7]. It is known that HSP70 regulates the cellular apoptosis: 1) directly, by intervening to the function of some proteins which induce the apoptotic cell death and 2) indirectly, by increasing the levels of anti-death protein bcl-2 [8]. All the previous findings converge to the protective role of HSP70.

In the present study, we sought to localize indicative cellular metabolic dysfunctions in the cerebellum of post mortem brains of schizophrenics and normal controls, using HSP70 as a biomarker of cellular metabolic alterations and a measure of neuronal injury in the brain [9].

\section{Methods and Materials}

\subsection{Characteristics of Human Subject Tissues}

The post mortem brain tissues from the region of cerebellar cortex were obtained from: 1) the Psychiatric Clinic of Bel Air University, Geneva and 2) the Forensic Pathology Service of Athens, Ministry of Justice. In total 
22 samples were included in our study.

In specific, the brain tissues from Bel Air University were obtained with medical and psychiatric records of the subjects and were diagnosed according to DSM-IV criteria: 10 cases of schizophrenia and 3 cases of controls. 9 brain tissues from the Forensic Pathology Service of Athens were collected and after a detailed study of the autopsy reports and toxicology records were defined as normal controls. These subjects died from sudden cardiac arrest. The patient data including age, sex, diagnosis, post mortem interval, form of tissue and cause of death are listed in Table 1.

\subsection{Preparation of Sections}

All brain tissues had volume $3 \mathrm{~cm}^{3}$ and were fixed in neutral buffer formalin for seven days and then processed and embedded in paraffin to form blocks for the best preservation of the post mortem tissues. For all tissue samples, total sections of $5-7 \mu \mathrm{m}$ thickness were cut from these blocks and were mounted onto microscope slides until use. For each case, total tissue sections were stained with Luxol Fast Blue-Cresyl violet (LFB-Cr) [10]. This histological study was succeeded for the demonstration of myelin phospholipids, mitochondria and nucleolus (stained with a dark azure color) and proteins (stained with light blue color). Nucleic acids, Nissl bodies and RNA were stained with a violet color.

\subsection{Immunohistochemical Detection of HSP70}

Tissue sections from cerebellar cortex of 10 schizophrenics and 12 controls (as mentioned before) were processed for immunochemical detection of HSP70 protein. The sections were exposed to heat $\left(58^{\circ} \mathrm{C}-60^{\circ} \mathrm{C}\right)$ for 2 hours and incubation with $3 \%$ hydrogen peroxide after rehydration was followed. Blocking agent (DAKO, Denmark) was added on the tissues for $10 \mathrm{~min}$ to block the endogenous peroxidase activity. Next, sections were incubated for $18 \mathrm{~h}$ at $4^{\circ} \mathrm{C}$ with the primary polyclonal rabbit antibody anti-HSP70 (1:1000, DAKO). Biotinylated Peroxidase conjugated goat anti-rabbit Igs (DAKO) was used for $10 \mathrm{~min}$. The enzyme horseradish peroxidase (HRP, DAKO) was used as the visualization system and DAB (Boehringer Ingelheim, Germany) was used as the chromogen. Sections were counterstained with haematoxylin. Three washes with TBS buffer were performed between the previous steps.

The reactivity of HSP70 antibody was confirmed in human breast carcinoma tissue as positive control. The omission of primary HSP70 antibody in each cerebellar tissue section of controls served as a negative control.

Table 1. Data of subjects.

\begin{tabular}{|c|c|c|c|c|c|}
\hline No & Age/Sex & Diagnosis & Autolysis time & Form of Tissue & Cause of death \\
\hline 1 & $47 / \mathrm{M}$ & Control & $7.5 \mathrm{hr}$ & Fresh and formalin fixative & Myocardial infarction \\
\hline 2 & $65 / \mathrm{M}$ & Control & $12.5 \mathrm{hr}$ & Fresh and formalin fixative & Myocardial infarction \\
\hline 3 & $60 / \mathrm{F}$ & Control & $21.5 \mathrm{hr}$ & Fresh and formalin fixative & Myocardial infarction \\
\hline 4 & $57 / \mathrm{M}$ & Control & $12 \mathrm{hr}$ & Fresh and formalin fixative & Myocardial infarction \\
\hline 5 & $46 / \mathrm{M}$ & Control & $24 \mathrm{hr}$ & Fresh and formalin fixative & Myocardial infarction \\
\hline 6 & $88 / \mathrm{M}$ & Control & $7 \mathrm{hr}$ & Fresh and formalin fixative & Myocardial infarction \\
\hline 7 & $91 / \mathrm{F}$ & Control & $20 \mathrm{hr}$ & Fresh and formalin fixative & Aneurysm of ascending aorta \\
\hline 8 & $82 / \mathrm{F}$ & Control & $18 \mathrm{hr}$ & Fresh and formalin fixative & Myocardial infarction \\
\hline 9 & $52 / \mathrm{F}$ & Control & $13 \mathrm{hr}$ & Fresh and formalin fixative & Myocardial infarction \\
\hline 10 & $48 / \mathrm{M}$ & Control & $16 \mathrm{hr}$ & Fresh and formalin fixative & Myocardial infarction \\
\hline 11 & $52 / \mathrm{M}$ & Control & $11 \mathrm{hr}$ & Fresh and formalin fixative & Myocardial infarction \\
\hline 12 & $58 / \mathrm{M}$ & Control & $10 \mathrm{hr}$ & Fresh and formalin fixative & Myocardial infarction \\
\hline 13 & $54 / \mathrm{F}$ & Schizophrenia & $24 \mathrm{hr}$ & Fresh and formalin fixative & Myocardial infarction \\
\hline 14 & $64 / F$ & Schizophrenia & $19 \mathrm{hr}$ & Fresh and formalin fixative & Cardiopumonary arrest \\
\hline 15 & $67 / \mathrm{F}$ & Schizophrenia & $6 \mathrm{hr}$ & Fresh and formalin fixative & Acute pulmonary edema \\
\hline 16 & $73 / \mathrm{M}$ & Schizophrenia & $8 \mathrm{hr}$ & Formalin fixative & Myocardial infarction \\
\hline 17 & $82 / \mathrm{M}$ & Schizophrenia & $15.5 \mathrm{hr}$ & Formalin fixative & Cardiopumonary arrest \\
\hline 18 & $94 / \mathrm{F}$ & Schizophrenia & $19 \mathrm{hr}$ & Formalin fixative & Myocardial infarction \\
\hline 19 & $47 / \mathrm{M}$ & Schizophrenia & $12 \mathrm{hr}$ & Formalin fixative & Myocardial infarction \\
\hline 20 & $68 / \mathrm{M}$ & Schizophrenia & $15 \mathrm{hr}$ & Formalin fixative & Myocardial infarction \\
\hline 21 & $55 / \mathrm{M}$ & Schizophrenia & $18 \mathrm{hr}$ & Formalin fixative & Myocardial infarction \\
\hline 22 & $73 / \mathrm{M}$ & Schizophrenia & $11 \mathrm{hr}$ & Formalin fixative & Acute pulmonary edema \\
\hline
\end{tabular}

M: Male, F: Female. 
All the tissue sections were scored on a scale ranging from 0 to 4 according to the intensity of the reaction. The subjective scores were determined by two observers.

\subsection{Protein Extraction and Western Blot Analysis}

$0.2 \mathrm{~g}$ of the brain fresh sections from 5 controls and 3 schizophrenics were homogenized in $2 \mathrm{ml}$ homogenization buffer ( $\mathrm{pH}$ 7.5): $50 \mathrm{mM}$ Tris hydroxymethyl aminomethane (Merck, Germany), $1 \mathrm{mM}$ EDTA (Bio-Rad, USA), 1\% Sodium deoxycholic acid (Serva, Germany), $0.5 \mathrm{M}$ Potassium chloride (Serva) and $13 \mu \mathrm{l} / \mathrm{lt}$ cocktail inhibitors (Sigma, Germany). The homogenization mixture was centrifuged at $2000 \mathrm{rpm}$ for $10 \mathrm{~min}$ at $4^{\circ} \mathrm{C}$. The sediment was collected and centrifuged at 14,000 rpm for $30 \mathrm{~min}$ at $4^{\circ} \mathrm{C}$. Supernatant containing total protein extracts were recovered and protein concentrations measured by the Bradford method, Bradford MM, 1976. After denaturation at $95^{\circ} \mathrm{C}$ for $5 \mathrm{~min}$, protein extracts $(50 \mu \mathrm{g}$ of total protein) and kaleidoscope prestain standard markers (positive control, Bio-Rad) were subjected to SDS/PAGE ( $10 \%$ polyacrylamide) at 100 volts for 2 hours at room temperature (RT) and transferred to pure nitrocellulose membrane (Bio-Rad) at 30 volts for 18 hours at $4^{\circ} \mathrm{C}$ using a mini Trans-blot electrophoretic transfer cell (BioRad).

The nitrocellulose membrane was treated with blocking solution: TBS buffer, $0.5 \%$ Tween 20 and $5 \%$ non-fat milk, for 1 hour at RT, then incubated for 18 hours at $4{ }^{\circ} \mathrm{C}$ with the primary polyclonal rabbit antibody anti-HSP70 (1:1000, DAKO) diluted in TBS buffer, $0.5 \%$ Tween 20 and $1 \%$ bovine serum albumin. Next, the membranes were incubated for 1 hour at RT with HRP conjugated secondary antibody (DAKO) diluted 1:2000 in TBS buffer, $0.5 \%$ Tween 20 and $1 \%$ non-fat milk. Three washes with TBS buffer and $0.5 \%$ Tween 20 were performed between the previous steps. Western blot was developed using the ECL detection kit (Amersham, Germany) and exposed to high performance chemiluminescence film (Kodak, USA). The quantification analysis was performed based on the band density data calculated with Gel Analyzer 3.0 software (Media Cybernetics, L.P., USA). In order to check the linearity of the band densities obtained by the chemiluminescence methodology, a Western blot assay without the primary antibody was performed (negative control) as above.

\subsection{Statistical Analysis}

Student's t-test analysis was applied to determine whether the differences observed between groups (schizophrenia and control) were statistically significant $(\mathrm{p}<0.05)$. For immunolabelling intensity, 3 microscopic areas in each layer of the cerebellar cortex: Molecular, Purkinje and
Granular $(20 \times, 40 \times$ and $100 \times$ magnification) were examined for each slide.

Differences in data obtained from the Western blot were also evaluated using Student's t-test.

\section{Results}

\subsection{Protein Distribution}

Cellular localization of HSP70 was revealed by immunohistochemistry. For the controls, immunohistochemistry images of the analysed areas showed moderate to intense immunoreactivity in the cytoplasm and dendrites of Purkinje cells (Figure 1(a)) whereas HSP70 immunoreactivitity (HSP70-IR) was intense in the synaptic glomeruli of the granule cell layer (Figure 1(c)). In the molecular layer, intense HSP70-IR was observed in the cytoplasm of the stellate cells (not shown). Compared with the controls, remarkable differences of reduced HSP70-IR were found in the group of schizophrenic samples. Specifically, mild HSP70-IR was marked in the cytoplasm of Purkinje cells (Figure 1(b)), whereas mild or no HSP70-IR was found in the synaptic glomeruli of the granule cell layer (Figure 1(d)). Lack of HSP70-IR was observed in the stellate cells (not shown).

The antibody used for the detection of HSP70 resulted in specific cytoplasmic staining in the human breast carcinoma sections (positive controls). However, the negative controls did not show any immunohistochemical reaction. Finally, many areas of synaptic glomeruli in the schizophrenics' cerebella showed lack of HSP70 immunoreactivity compared to the controls $(\mathrm{p}<0.01)$. The Mean \pm SEM score, ranging from 0 to 4 according to the intensity of the reaction, for the controls was $2.92 \pm 0.23$ and for the schizophrenics was $1.30 \pm 0.21$.

\subsection{Expression of HSP70}

Tissue extracts from the cerebellum were subjected to Western blot analysis to examine quantitative changes in the expression of HSP70 in control and schizophrenic groups. A single band of $70 \mathrm{kDa}$, approximately, was obtained by Western blotting, demonstrating the specificity of the anti-HSP70 antibody. Statistically significant differences were observed between controls and schizophrenics with respect to global HSP70 expression in the single CNS zone (Figure 2(a)). The optical density of controls' protein bands was almost twice higher than the schizophrenics' protein bands $(\mathrm{p}=0.046)($ Figure 2(b)).

\section{Discussion}

The main findings of the present study are that: 1) HSP70 is expressed in the synaptic glomeruli and in the cytoplasm and dendrites of Purkinje cells, of the normal controls' cerebellar cortex; 2) cerebellar HSP70, at the 


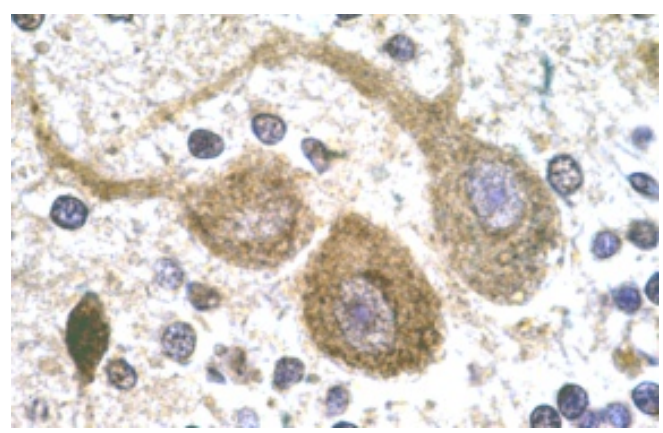

(a)

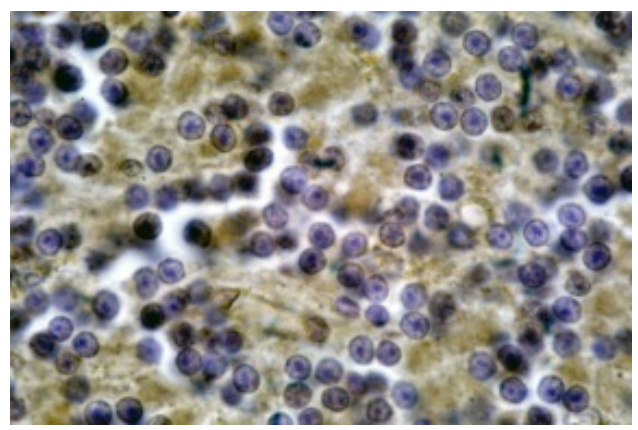

(c)

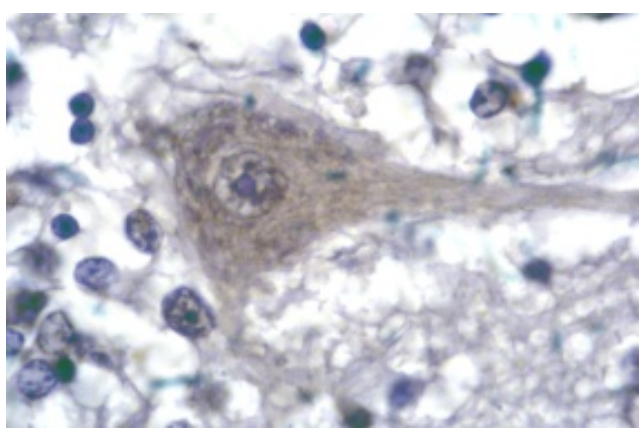

(b)

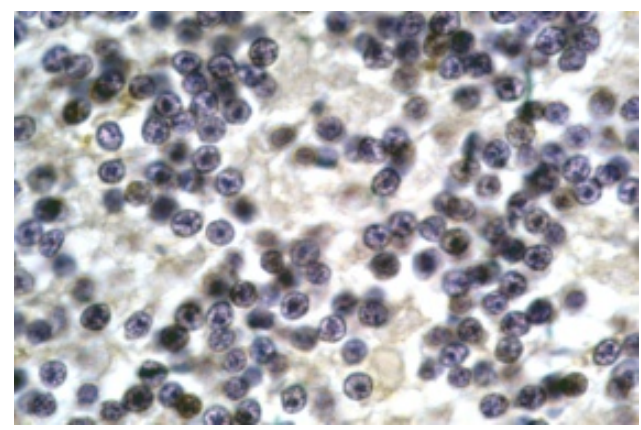

(d)

Figure 1. Representative sample of photos. Purkinje neurons of control subject showing HSP70 IR deposits in neuronal cytoplasm and dendrites as well as dense accumulations in nuclear cups (a). Purkinje neuron of schizophrenic subject showing weak cytoplasmic HSP70 IR but moderate reaction in cytoplasmic aggregates (b). Intense HSP70 IR in the synaptic glomeruli of the granule cell layer (c). Absence of HSP70 IR in the areas of synaptic glomeruli of the granule cell layer (d). Anti-HSP70/DAB/Gill's haematoxylin. × 1150.

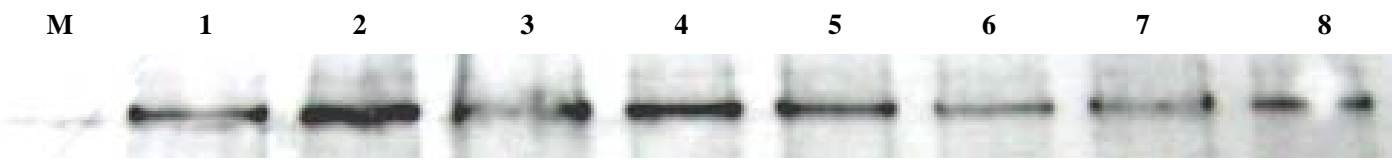

M: Marker, 1-5: Controls, 6-8: Schizophrenics

(a) Western blot

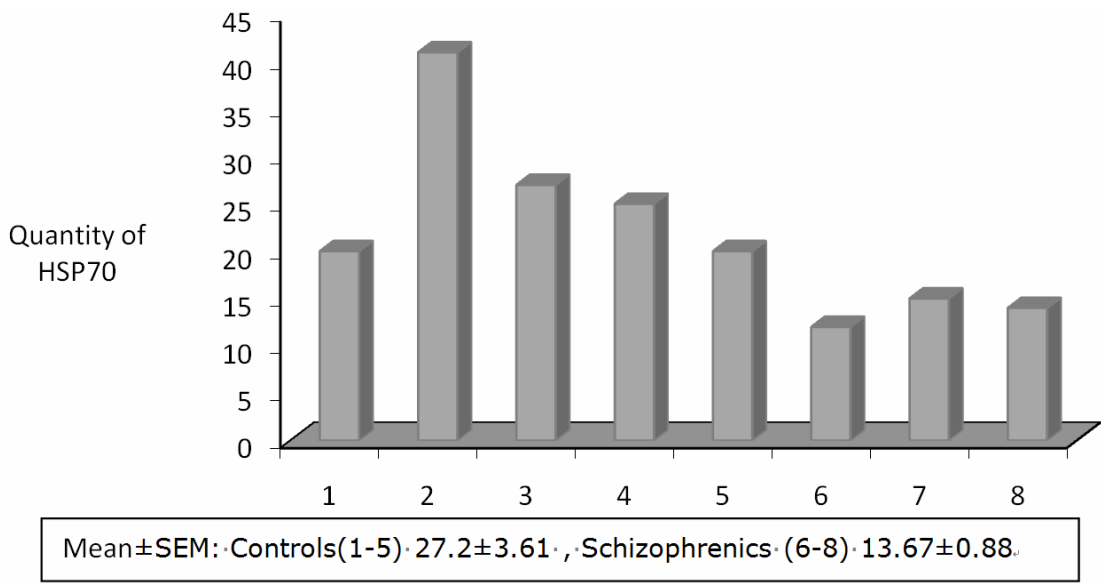

(b) Optical Density

Figure 2. (a) A blot of 15\% polyacrylamide gel probed with the anti-HSP70 antibody (single band). Lanes 1 - 5 were samples of cerebellar human controls. Lanes 6 - 8 were samples of cerebellar human schizophrenics. Lane M contained prestained molecular weight standard (Bio Rad, Hercules, CA), whose size is 70 Kilodaltons; (b) The Diagram represents the total amount of HSP70 protein (in each sample), resulting from the measurement of optical density of single bands that are shown in Figure 2(a). 
same areas, is significantly reduced in schizophrenia. In a study [11], the levels of HSPA 12A (member of family of protein stress HSPs which shows a strong domain homology to HSP70) were low in the dorsolateral prefrontal cortex (DLPFC) of schizophrenic patients compared to normal controls. In addition, at the same study, the levels of HSPA 12A on the DLPFC of subjects with Major Depressive Disorder showed no change compared with the controls. This suggests that the expression change observed in schizophrenia is not characteristic of all brain disorders. It is known that the overexpression of HSP70 in vivo protects the brain from stressful factors as ischemia and heat shock that cause damage [12].

The cause of death of all the control subjects in our study was the cardiac arrest that resulted to global cerebral ischemia which induces the expression of HSP70 and protect brain cells from damage [13,14]. Thus, the cardiac arrest is the one of the causes of death that establish subjects as normal controls in order to compare them with pathological subjects.

It is interesting to note that all patients of our study had received antipsychotics. This fact raises the question as to whether this medication could have caused HSP70 reduction. The cerebellum is probably not the target of antipsychotics since most of its neurochemical and morphological sequelae occur in the striatum and prefrontal cortex. Antipsychotics do not affect the expression of synaptic proteins in the cerebellum [15]. On the other hand, in a study of schizophrenic patients without medication, a frequency of patients showed high levels of HSP70 antibodies whereas after six weeks of antipsychotic treatment the levels were decreased significantly [16]. In another similar study of HSP70 antibodies in patients with schizophrenia, six out of seven drug-free patients experiencing their first episode of schizophrenia showed high antibody titers against HSP70 [17]. As the author suggests, this finding may be an important mechanism in a subgroup of patients with schizophrenia.

Reduced or lacking of HSP70 immunoreaction in the cerebellar synaptic glomeruli of schizophrenic patients reveals abnormal synaptic organization that contributes to neurological dysfunction associated with schizophrenia. Schizophrenia is a heterogeneous disease that encompasses different phenotypic manifestations of the disease. Different subjects with schizophrenia within a sub-class have different subject-specific synaptic deficits, but they all converge at a functional level to affect synaptic transmission between neurons. Synaptic disturbances in schizophrenia cannot be studied and understood as an independent disease hallmark, but only as a part of a complex network of events. In a recent study, colocalization of DISC1 and HSP70, in both cell lines and neurons was reported. However, it was showed that by enhancing proteasomal activity the DISC1 aggresome formation was reduced and the aggresome was processed by fusion with the autophagosome [18]. This may be a possible explanation of the reduced or absence HSP70 immunoreactivity in the cerebellar synaptic glomeruli of schizophrenic patients in our study. The synaptic pathology of cerebellum in schizophrenia rather selectively acts on the excitatory neurons, i.e. granule cells [19]. In a study, ablation of Golgi cells in the cerebellum reduced the release of GABA ( $\gamma$-aminobutyric acid) that caused the excitability of NMDA (N-methyl D-aspartate) glutamate receptors in granule cells [20]. Another study investigating cerebellum of patients with schizophrenia and depression showed reduced levels of the enzyme glutamic acid decarboxylase that is responsible for the conversion of glutamate to $\gamma$-aminobutyric acid [21]. Moreover, the cerebellum is involved in many activities of the cerebral cortex. Thus any abnormality of the cerebellum can lead to different dysfunctions of the cerebral cortex, i.e. cognitive, and consequently results to a variety of pathological symptoms as observed in schizophrenia. The reduced levels or absence (in many synaptic glomeruli) of HSP70 may also affect the transfer of information from granular to Purkinje cells. These two types of cells, synergistically, contribute to the setting or synchronization of cerebral cortex, providing information to nuclei such as dentate nucleus [22].

There are some limitations to the current study including the small sample size of post mortem brains for immunohistochemistry and the even smaller sample size for Western blot analysis. This study (as all post mortem studies) offers very limited insight into the time course by which the synaptic alterations concerning HSP70 develop in schizophrenia.

The present study showed the dysfunction of synapses in the cerebellar glomeruli of the granular layer, in patients with schizophrenia, by reduced levels or absence of HSP70. This finding supports: 1) the synaptic pathology and 2) abnormality of neuroprotective mechanisms in neuronal networks of the cerebellum in schizophrenia.

\section{Acknowledgements}

This work was supported by the Theodor Theohari Cozzica Foundation. The author thanks the Psychiatric Clinic of Bel Air University, Geneva and Soultana Marianou in the Forensic Pathology Service of Athens, Ministry of Justice, for providing the human brain tissues. Special thanks to Olga Manthou for her technical support. The author declares no conflicts of interest.

\section{REFERENCES}

[1] N. C. Andreasen, "A Unitary Model of Schizophrenia," Archives of General Psychiatry, Vol. 56, No. 9, 1999, pp. 781-787. doi:10.1001/archpsyc.56.9.781 
[2] H. Picard, I. Amado, S. Mouchet-Mages, J. P. Olie and M. O. Krebs, "The Role of the Cerebellum in Schizophrenia: An Update of Clinical, Cognitive, and Functional Evidences," Schizophrenia Bulletin, Vol. 34, No. 1, 2008, pp. 155-172. doi:10.1093/schbul/sbm049

[3] D. O. Frost, C. A. Tamminga, D. R. Medoff, C. Caviness, G. Innocenti and W. T. Carpenter, "Neuroplasticity and Schizophrenia," Biological Psychiatry, Vol. 56, No. 8, 2004, pp. 540-543. doi:10.1016/j.biopsych.2004.01.020

[4] S. L. Eastwood and P. J. Harrison, "Synaptic Pathology in the Anterior Cingulate Cortex in Schizophrenia and mood disorders. A review and A Western Blot Study of Synaptophysin, GAP-43 and the Complexins," Brain Research Bulletin, Vol. 55, No. 5, 2001, pp. 569-578. doi:10.1016/S0361-9230(01)00530-5

[5] M. L. Phillips, W. C. Drevets, S. L. Rauch and R. Lane, "Neurobiology of Emotion Perception II: Implications for Major Psychiatric Disorders," Biological Psychiatry, Vol. 54, No. 5, 2003, pp. 515-528. doi:10.1016/S0006-3223(03)00171-9

[6] A. Parcellier, S. Gurbuxani, E. Schmitt, E. Solary and C. Garrido, "Heat Shock Proteins, Cellular Chaperones that Modulate Mitochondrial Cell Death Pathways," Biochemical and Biophysical Research Communications, Vol. 304, No. 3, 2003, pp. 505-512. doi:10.1016/S0006-291X(03)00623-5

[7] R. G. Giffard and M. A. Yenari, "Many Mechanisms for Hsp70 Protection from Cerebral Ischemia," Journal of Neurosurgical Anesthesiology, Vol. 16, No. 1, 2004, pp. 53-61. doi:10.1097/00008506-200401000-00010

[8] S. Takayama, J. C. Reed and S. Homma, "Heat-Shock Proteins as Regulators of Apoptosis," Oncogene, Vol. 22, No. 56, 2003, pp. 9041-9047. doi:10.1038/sj.onc.1207114

[9] J. Q. Lan, J. Chen, F. R. Sharp, R. P. Simon and S. H. Graham, "Induction of Heat-Shock Protein (HSP72) in the Cingulated and Retrosplenial Cortex by Drugs that Antagonize the Effects of Excitatory Amino Acids," Brain Research Molecular Brain Research, Vol. 46, No. 1-2, 1997, pp. 297-302. doi:10.1016/S0169-328X(97)00003-X

[10] H. Kluever and E. Barrera, "A Method for the Combined Stainning of Cells and Fibres in the Nervous System," Journal of Neuropathology, Vol. 12, No. 4, 1953, pp. 400-403. doi:10.1097/00005072-195312040-00008

[11] J. L. Pongrac, F. A. Middleton, L. Peng, D. A. Lewis, P. Levitt and K. Mirnics, "Heat Shock Protein 12A Shows Reduced Expression in the Prefrontal Cortex of Subjects with Schizophrenia," Biological Psychiatry, Vol. 56, No. 12, 2004, pp. 943-950. doi:10.1016/j.biopsych.2004.09.005

[12] F. R. Sharp, S. M. Massa and R. A. Swanson, "HeatShock Protein Protection," Trends in Neurosciences, Vol. 22, No. 3, 1999, pp. 97-99. doi:10.1016/S0166-2236(98)01392-7
[13] M. A. Yenari, R. M. Sapolsky and G. K. Steinberg, "The Neuroprotective Potential of Heat Shock Protein 70 (HSP70)," Molecular Medicine Today, Vol. 5, No. 12, 1999, pp. 525-531. doi:10.1016/S1357-4310(99)01599-3

[14] I. R. Brown, "Heat Shock Proteins and Protection of the Nervous System," Annals of the New York Academy of Sciences, Vol. 1113, 2007, pp. 147-158. doi:10.1196/annals.1391.032

[15] S. L. Eastwood, A. J. Law, I. P. Everall and P. J. Harrison, "The Axonal Chemorepellant Semaphorin 3A is Increased in the Cerebellum in Schizophrenia and May Contribute to Its Synaptic Pathology," Molecular Psychiatry, Vol. 8, No. 2, 2003, pp. 148-155. doi:10.1038/sj.mp.4001233

[16] J. J. Kim, S. J. Lee, K. Y. Toh, C. U. Lee, C. Lee and I. H. Paik, "Identification of Antibodies to Heat Shock Proteins $90 \mathrm{Kda}$ and $70 \mathrm{Kda}$ in Patients with Schizophrenia," Schizophrenia Research, Vol. 52, No. 1, 2001, pp. 127-135. doi:10.1016/S0920-9964(00)00091-8

[17] M. J. Schwarz, M. Riedel, R. Gruber, M. Ackenheil and N. Muller, "Antibodies to Heat Shock Proteins in Schizophrenic Patients: Implications for the Mechanism of the Disease," American Journal of Psychiatry, Vol. 156, No. 7, 1999, pp. 1103-1104.

[18] T. A. Atkin, N. J. Brandon and J. T. Kittler, "Disrupted in Schizophrenia 1 Forms Pathological Aggresomes that Disrupt Its Function in Intracellular Transport," Human Molecular Genetics, Vol. 21, No. 9, 2012, pp. 2017-2028. doi: $10.1093 / \mathrm{hmg} / \mathrm{dds} 018$

[19] S. L. Eastwood, D. Cotter and P. J. Harrison, "Cerebellar Synaptic Protein Expression in Schizophrenia," Neuroscience, Vol. 105, No. 1, 2001, pp. 219-229. doi:10.1016/S0306-4522(01)00141-5

[20] D. Watanabe, H. Inokawa, K. Hashimoto, N. Suzuki, M. Kano, R. Shigemoto, T. Hirano, K. Toyama, S. Kaneko, M. Yokoi, K. Moriyoshi, M. Suzuki, K. Kobayashi, T. Nagatsu, R. J. Kreitman, I. Pastan and S. Nakanishi, “Ablation of Cerebellar Golgi Cells Disrupts Synaptic Integration Involving GABA Inhibition and NMDA Receptor Activation in Motor Coordination," Cell, Vol. 95, No. 1, 1998, pp. 17-27. doi:10.1016/S0092-8674(00)81779-1

[21] S. H. Fatemi, J. M. Stary, J. A. Earle, M. Araghi-Niknam and E. Eagan, "GABAergic Dysfunction in Schizophrenia and Mood Disorders as Reflected by Decreased Levels of Glutamic Acid Decarboxylase 65 and $67 \mathrm{Kda}$ and Reelin Proteins in Cerebellum," Schizophrenia Research, Vol. 72, No. 2, 2005, pp. 109-122. doi:10.1016/j.schres.2004.02.017

[22] N. C. Andreasen and R. Pierson, "The Role of the Cerebellum in Schizophrenia," Biological Psychiatry, Vol. 64, No. 2, 2008, pp. 81-88. doi:10.1016/j.biopsych.2008.01.003 